\title{
Organisation, Systematik und Biologie von Microbedyle cryptophthalma nov. spec. (Gastropoda, Opisthobranchia) aus dem Brandungsstrand des Mittelmeeres
}

\author{
W. WESTHEIDE ${ }^{1} \&$ E. WAWRA ${ }^{2}$ \\ 11. Zoologisches Institut der Universität Göttingen; \\ Göttingen, Bundesrepublik Deutschland \\ und \\ 21II. Zoologische Abteilung, Naturbistorisches Museum; \\ Wien, Österreich
}

\begin{abstract}
Organization, taxonomy and biology of Microbedyle cryptopbtbalma nov. spec. (Gastropoda, Opisthobranchia) from sandy surf-beaches of the Mediterranean Sea. Microbedyle cryptophthalma noy. spec. was found in the mesopsammon of different sandy beaches of the Mediterranean Sea (Tunisia, Italy, France and Lebanon). The slugs inhabit a small, particularly exposed region of the surf-beach generally covered by waves except in the sampling locality in Italy where maximum abundance occured about $2 \mathrm{~m}$ above the surfedge. The vertical distribution extends down to a substrate depth of $15 \mathrm{~cm}$. Distribution was studied as a function of average sediment particle size, but no definite relationship could be established. The new species is placed in the family Microhedylidae because of its size, external morphology, male sexual organs, form of the tentacles and shape of the midgut gland. The epidermal enclosures are almost identical to those described by KowaLEvSKY (1901) in Parbedyle tyrtowii. But Microbedyle cryptopbtbalma is a clearly distinct species by the formula of the asymmetrical radula $(1.1 .2 \times \mathrm{n})$, the form of the teeth, and the almost invisible eyes. Parhedyle is a junior synonym of Microbedyle and must be eliminated. The only species, $P$. tyrtowii, shows no significant differences and is, therefore, considered to belong to the genus Microhedyle. Sexes in $M$. cryptophthalma are separate; sperm are transferred through simple spindle-shaped spermatophores. Spermatophores, body size, extremely small eyes, and lack of pigment are recognized as typical "Lebensform"-characteristics of the interstitial sand fauna.
\end{abstract}

\section{EINLEITUNG}

Gastropoden gehören $z \mathfrak{u}$ den interessantesten Organismen der interstitiellen Fauna. Überkommenen Merkmalen der Molluskenorganisation stehen bei dieser Gruppe viele Anpassungsmerkmale an den Lebensraum Mesopsammal gegenüber. Dieses gilt vor allem für die Ordnung der Acochlidiacea, deren Vertreter bei geringen Körperdimensionen den Habitus typischer Gastropoden bewahrt haben. 
Lange vor der eigentlichen Entdeckung der Lebensgemeinschaft der Sandlückenfauna beschrieb Kowalevsky (1901) die ersten Arten aus dieser Ordnung. Zur weiteren Kenntnis der interstitiellen Gastropoden haben dann besonders HerTLING (1930), Odhner $(1937,1952)$, Marcus (1953), Marcus \& Marcus $(1954,1955)$, Swedmark (1968a), Challis (1970) und Salvini-Plawen (1973) beigetragen. Eidonomie und Anatomie wurden durch diese Arbeiten weitgehend aufgeklärt, dagegen sind unsere Kenntnisse über die Biologie und Okologie weitaus geringer (SwEDMARK 1968b; Challis 1969a, 1969b; Hadl et al. 1970; Poizat 1971).

Am häufigsten sind die Acochlidiacea in den Lebensgemeinschaften sublitoraler Schill- und Sandböden (u. a. Salvini-Plawen 1968, 1973). Die vorliegende neue Art gehört dagegen zur Fauna der Brandungsstrände und ist weit verbreitet im Bereich des Mittelmeeres.

Der Artname der Microbedyle cryptophtbalma nov. spec. bezieht sich auf die schwer zu erkennenden Augen der Tiere.

\section{MATERIAL UND METHODIK}

$50 \mathrm{ccm}$ Sandproben wurden nach der allgemein im II. Zoologischen Institut in Göttingen angewandten Methodik (SснміDт 1968) aus dem Strand entnommen. Die Tiere sind dann mit einer nach der UHLIG-Methode arbeitenden Apparatur (WESTHEIDE \& SCHMIDT 1969) extrahiert worden. Insgesamt wurden etwa 170 Exemplare gefunden, von denen über 35 in lebendem Zustand (Phasenkontrastoptik) und 12 als histologische Präparate ( $3 \mu \mathrm{m}$ - Schnittsenien; Bouin-Fixierung, Azan-Färbung) untersucht werden konnten.

\section{FUNDORTE UND OKOLOGISCHE DATEN}

Microbedyle cryptophthalma wurde zuerst bei ökologisch-systematischen Untersuchungen in einem Sandstrand der tunesischen Mittelmeerküste, Golf von Tunis, Amilcar, gefunden (Juli 1969, März 1970, September 1971). Genauere Angaben über diese Probestelle können aus WeSTHEIDE (1970) entnommen werden. Weitere Fundorte an der tunesischen Küste sind die Insel Djerba, Südtunesien, nördlicher Strand unterhalb des Hotels Ulysse (März 1971) und Sandstrände bei Nabeul und Hammamet, Golf von Hammamet (Juli 1969, September 1971). Bei einem Aufenthalt an der Zoologischen Station Neapel (WeSTHEIDE, September 1970) wurde die Art an der Küste nördlich der Stadt bei Arco Felice und Castelvolturno nachgewiesen. Fixierte Exemplare (leg. Prof. P. Ax, 1967) liegen vom Sandstrand von Canet-Plage, Frankreich, vor. 18 fixierte Individuen aus Sandstränden des Libanons wurden uns von Dr. N. C. Hulings zur Verfügung gestellt.

In den tunesischen Stränden besiedeln die Tiere ein eng begrenztes Areal von 2 bis $3 \mathrm{~m}$ Breite innerhalb der Brandungszone, in einer Region, die noch dauernd von den Wellen erreicht wird. Nur bei Arco Felice (Italien) lag das Abundanzmaximum etwa $2 \mathrm{~m}$ über der Brandungskante; aber auch hier war der Sand noch vollständig wasser- 
gesättigt. Die vertikale Ausdehnung der Population reicht nur bis in $15 \mathrm{~cm}$ Tiefe; die unmittelbare Oberfläche wird gemieden.

Diese verschiedenen Strände besitzen teilweise recht unterschiedliche Sedimente (Abb. 1) (MD-Werte zwischen 325 und $825 \mu \mathrm{m}$, Sortierungskoeffizienten zwischen 1,24 und 1,64). Die Korngrößenverteilung hat daher wohl für das Auftreten der Art und ihre Verteilung keine große Bedeutung. Die Salzgehaltswerte entsprechen denen des freien Wassers.

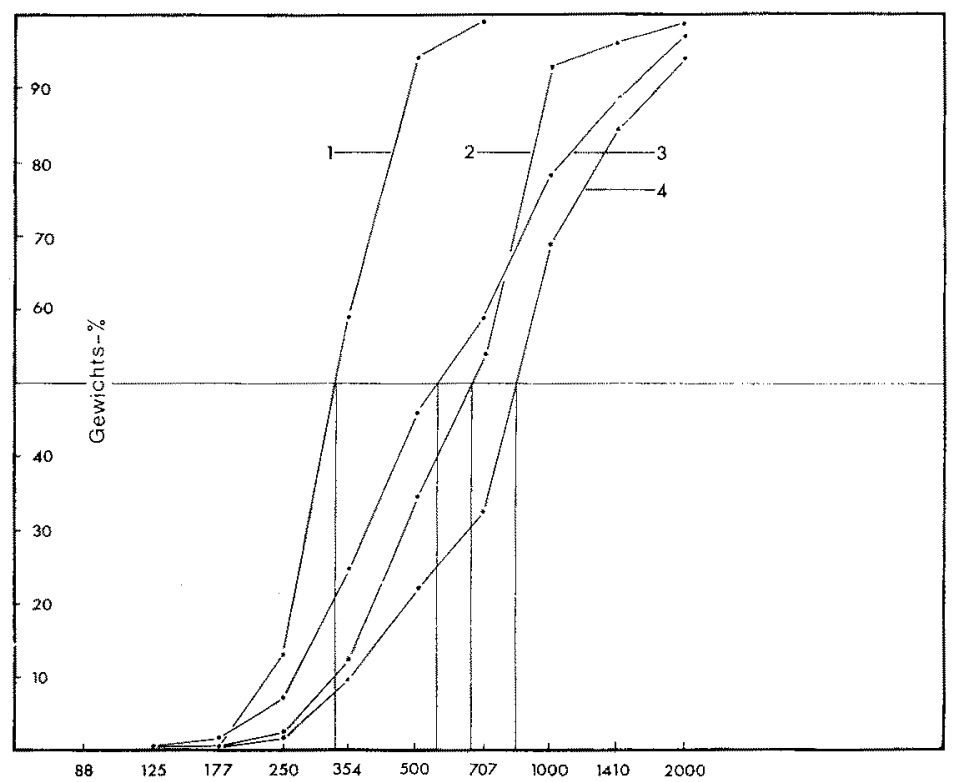

Abb. 1: Korngrößenverteilung von 4 ausgewählten Proben, in denen Microbedyle cryptophthalma gefunden wurde. (1) Castelvolturno, Italien; (2) Nabeul, Tunesien; (3) Amilcar, Tunesien; (4) Hammamet, Tunesien. Die senkrechten Linien geben die MD-Werte an

Die Abundanzwerte an der tunesischen Küste, besonders bei Amilcar, waren gering; mehr als 10 Tiere in $50 \mathrm{ccm}$ Sand wurden niemals gefunden. In Proben von Arco Felice konnten dagegen bis zu 23 Individuen in $50 \mathrm{ccm}$ ausgezählt werden.

Charakteristische Arten der Begleitfauna sind im Strand von Amilcar (Tunesien) die Gastrotrichen Chaetonotus aculifer Gerlach, Paraturbanella teissieri Swedmark, Tetranchyroderma papii GerLach, der Polychaet Hesionides arenaria Friedrich und der Tardigrade Batillipes pennaki Marcus.

\section{ORGANISATION}

Der Körper von Microbedyle cryptopbthalma gliedert sich in einen halsartigen Teil mit Kopf und einen deutlich breiteren Eingeweidesack (Abb. 2). Am halsartigen Teil ist auf der Ventralscite ein schmaler Fuß abgegliedert, der bis zur Mitte des Ein- 
geweidesackes reicht (Abb. 7). Vom Kopf bis zum distalen Ende des Fußes verläuft eine etwa $25 \mu \mathrm{m}$ breite Wimpersohle, mit der die Tiere sich ständig gleitend fortbewegen. Fußdrüsen, die innerhalb und außerhalb der Cilienzone münden, ermöglichen eine Festheftung am Substrat. Kopf und Hals-Fuß-Komplex können bei Beunruhigung

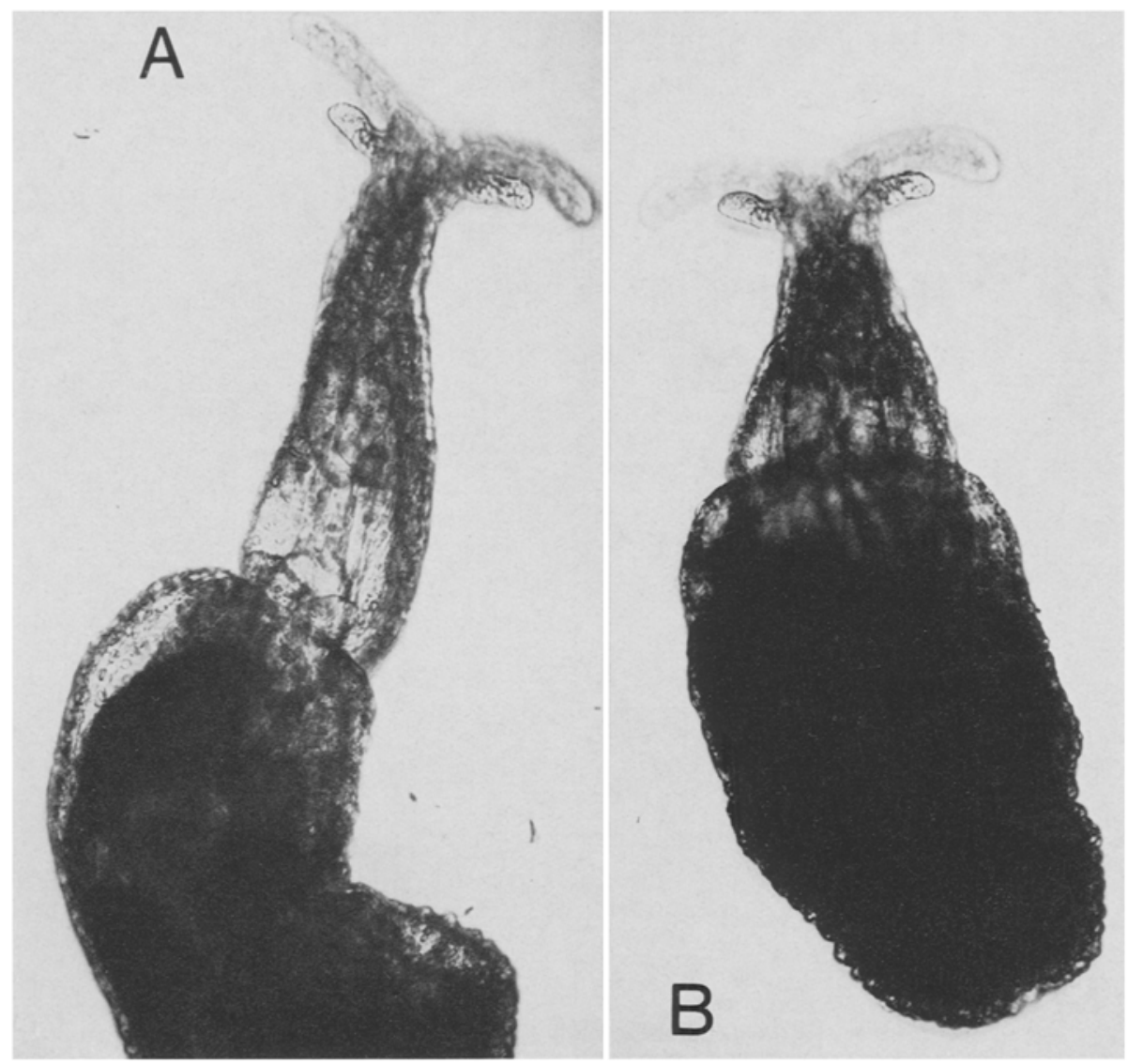

Abb. 2: Microhedyle cryptophtbalma nov. spec. A Habitusbild eines kriechenden Tieres; $B$ teilweise kontrahiertes Tier

vollständig in den hinteren Körperabschnitt zurüdkgezogen werden. Je nach Kontraktionszustand des Tieres beträgt die Breite des halsartigen Teiles 90 bis $180 \mu \mathrm{m}$, die des Eingeweidesackes 135 bis $460 \mu \mathrm{m}$. Die Körperlänge ist ebenfalls sehr variabel, so wurden Werte zwischen 700 und $1600 \mu \mathrm{m}$ bei verschiedenen Individuen gemessen.

Der Kopf besitzt zwei Paar Anhänge: die zylindrischen, meist flach liegenden Labialtentakel $(\mathrm{t})$ sind bei erwachsenen Tieren etwa $130 \mu \mathrm{m}$ lang; die schmalen, seitlich nach oben stehenden Rhinophoren ( $\mathrm{rh}$ ) besitzen eine Länge von etwa $75 \mu \mathrm{m}$. Die Kopfanhänge tragen kleine steife Tastcilien.

Die völlig pigmentlosen Tiere erscheinen im Auflicht weiß und fast undurchsichtig. 
Die Körperoberfläche ist durch unregelmäßige Falten eingeschnürt. An Quetschpräparaten erkennt man verschiedene Einlagerungen im Integument: (1) Ringförmige Einschlüsse, (2) plattenförmige Einschlüsse, (3) eiförmige, stark lichtbrechende Einschlüsse (Abb. 3 und 4).

Besonders charakteristisch für die Art sind die zahlreichen flachen ringförmigen Einschlüsse (1), die über den gesamten Körper verteilt liegen und auch in den Kopfanhängen zu finden sind (Durchmesser 8 bis $9 \mu \mathrm{m}$ ). Sie bestehen aus einer unterschiedlichen Zahl (etwa 14 bis 21) kleiner rechteckiger Glieder, die wie an einem Faden auf-

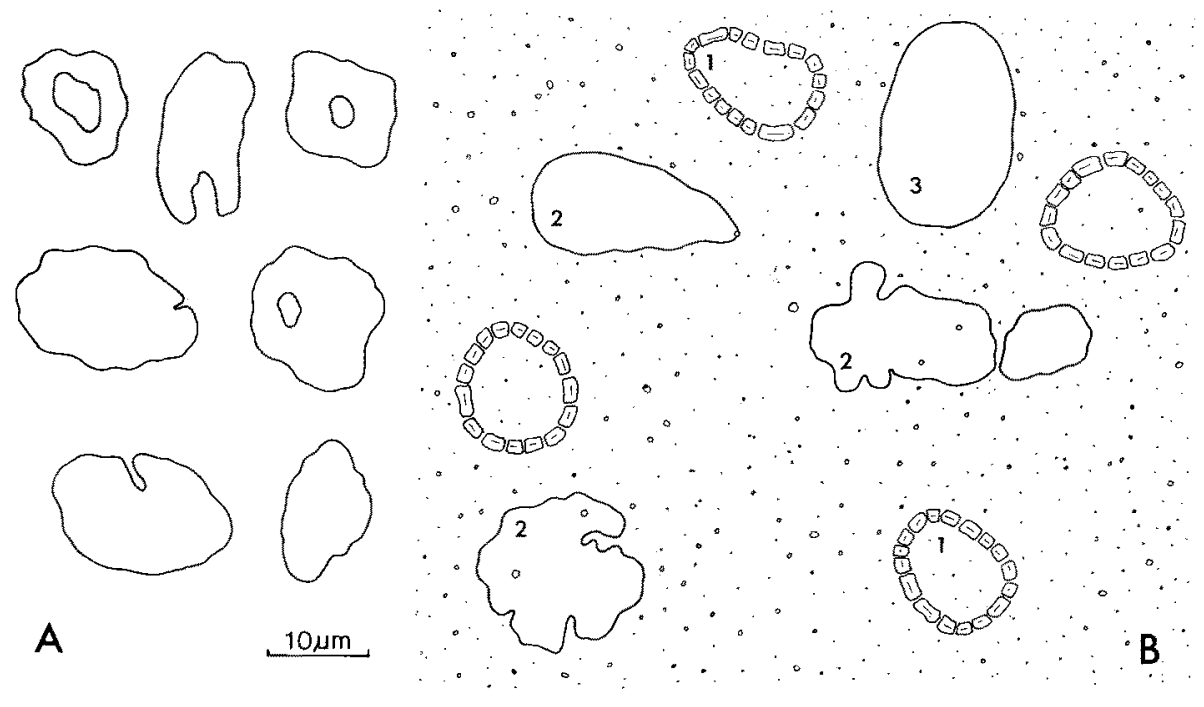

Abb. 3: Microhedyle cryptophthalma nov. spec. Einschlüsse im Integument. A plattenförmige Einschlïsse von Individuen, die bei Nabeul gefunden wurden. $B$ Einschlüsse von Individuen bei Amilcar; Quetschpräparat mit Zeichenapparat; (1) ringförmige Einschlüsse, (2) plattenförmige Einschlüsse, (3) eiförmige, stark lichtbrechende Einschlüsse

gereiht erscheinen. Bei starker Quetschung treten sie aus dem Körper aus; bei Zugabe verdünnter Essigsäure kann man erkennen, wie die Ringe aufreißen und die Glieder sich langsam auflösen. Es handelt sich daher wahrscheinlich um Kalkelemente. Sehr ähnliche Einlagerungen hat schon Kow ALEvsky (1901) bei Microbedyle tyrtowii (bezüglich der Nomenklatur vergleiche die Diskussion auf p. 37), als „corps en chapelet“ beschrieben; er deutete sie allerdings als Flüssigkeitströpfchen.

Von ungefähr gleicher Größe sind die plattenförmig erscheinenden Einschlüsse, die vor allem im Integument des Eingeweidesackes verteilt sind (2). Ihre Struktur kann bei den einzelnen Individuen unterschiedlich sein. Differenzen treten auch zwischen den Populationen verschiedener Fundorte auf. Bei den Amilcar-Tieren sind es runde oder ovale Gebilde (Durchmesser 10 bis $18 \mu \mathrm{m}$ ) mit unregelmäßigen, teilweise stark zerklüfteten Umrißlinien (Abb. 3B). Auf fast allen erkennt man ein oder mehrere stark lichtbrechende Punkte. Bei stark gequetschten Untersuchungsobjekten wölben diese Einschlüsse die Epidermis nach außen (Abb. 4D), und man sieht dann, daß es sich nicht 
um Platten, sondern um massive rundliche Konkremente handelt. Sie lösen sich bei Zugabe von Essigsäure auf.

Bei Individuen yon Nabeul finden sich neben diesen Gebilden auch solche, die eine mehr oder weniger große zentrale Durchbohrung besitzen ( $\mathrm{Abb} .3 \mathrm{~A}$ ). Sie ähneln den Einschlüssen, die KowAlevsKY (1901) bei Microhedyle tyrtowii als Kalkkörperchen beschrieben hat. Entsprechend, aber etwas regelmäßiger gestaltet, sind die Einlagerungen in den Individuen von Neapel.
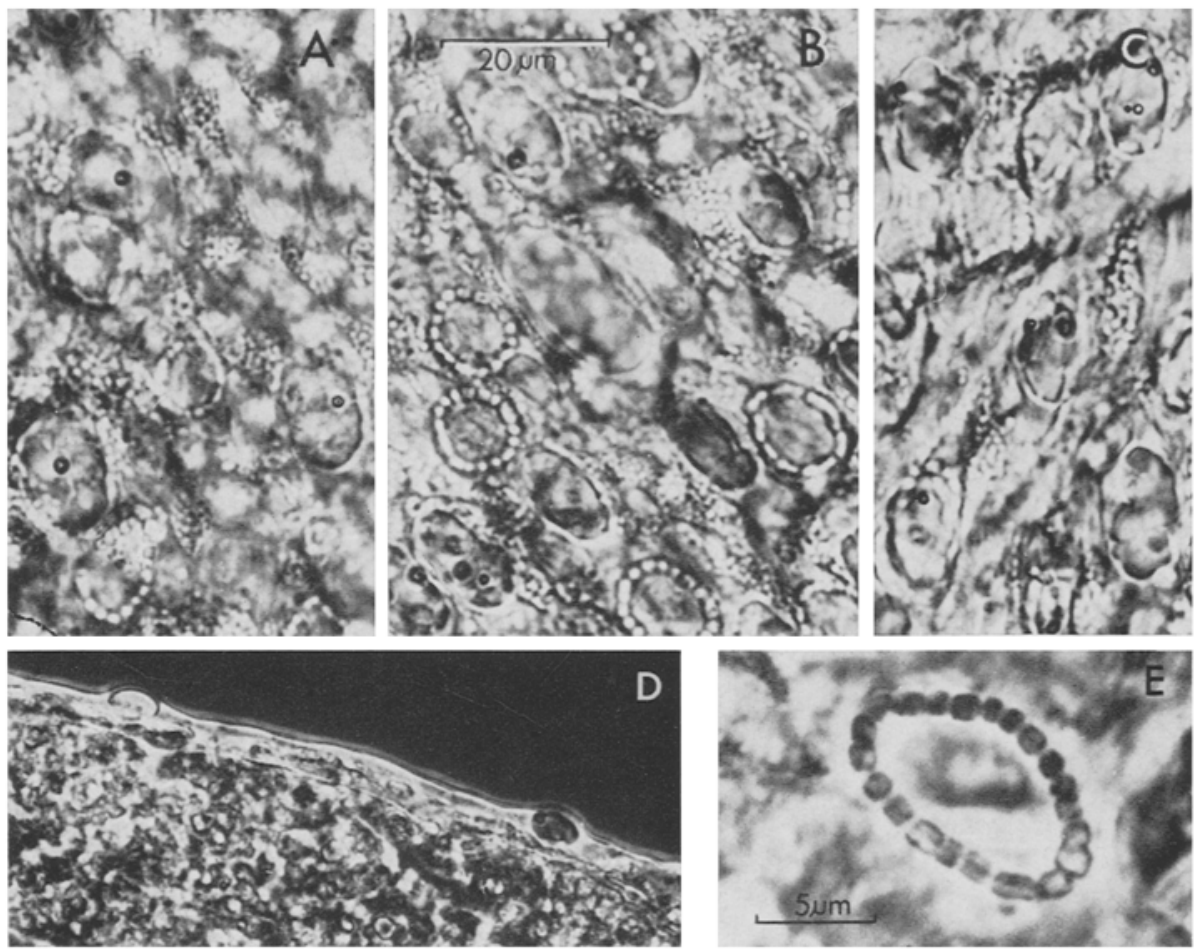

Abb. 4: Microbedyle cryptophthalma nov. spec. Einschlüsse im Integument; Individuen von Amilcar. $A, B, C$ ringförmige, plattenförmige und stark lichtbrechende Einschlüsse, $D$ stark gequetschtes Individuum mit plattenförmigen Einschlïssen, welche die Kutikula nach außen vorwölben, $E$ ringförmiger Einschluß (Größenangaben im Text)

Die dritte Art von Einschlüssen liegt meist im Bereich des Eingeweidesackes: wenige, stark lichtbrechende, eiförmige Gebilde (3). Sie unterscheiden sich von den vorher genannten Einlagerungen durch ihre Größe (etwa $20 \mu \mathrm{m}$ ) und ihre regelmäßigere Form (Abb. 3B). Man kann sie ebenfalls mit Essigsäure auflösen.

Die innere Organisation von Microbedyle cryptophtbalma zeigt keine signifikanten Unterschiede zu anderen Arten der Gattung (Kowalevsky 1901, Hertling 1930, Marcus \& Marcus 1954); die Beschreibung beschränkt sich daher auf die systematisch interessanten Merkmale.

Im cerebro-pedalen Komplex vor dem Pharynxbulbus liegt in den Pedalganglien 


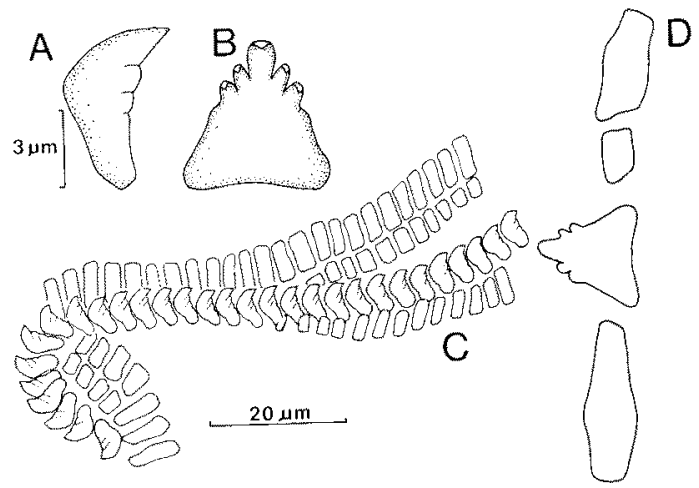

Abb. 5: Microbedyle cryptophthalma nov. spec. Radula. A Rhachiszahn, Seitenansicht; $B$ Rhachiszahn, Aufsicht; $C$ Radula, Quetschpräparat; die seitlichen Zahnreihen sind nur soweit gezeichnet, wie sie in diesem Präparat zu erkennen waren (mit Zeichenapparat); $D$ Anordnung der Zahnreihen

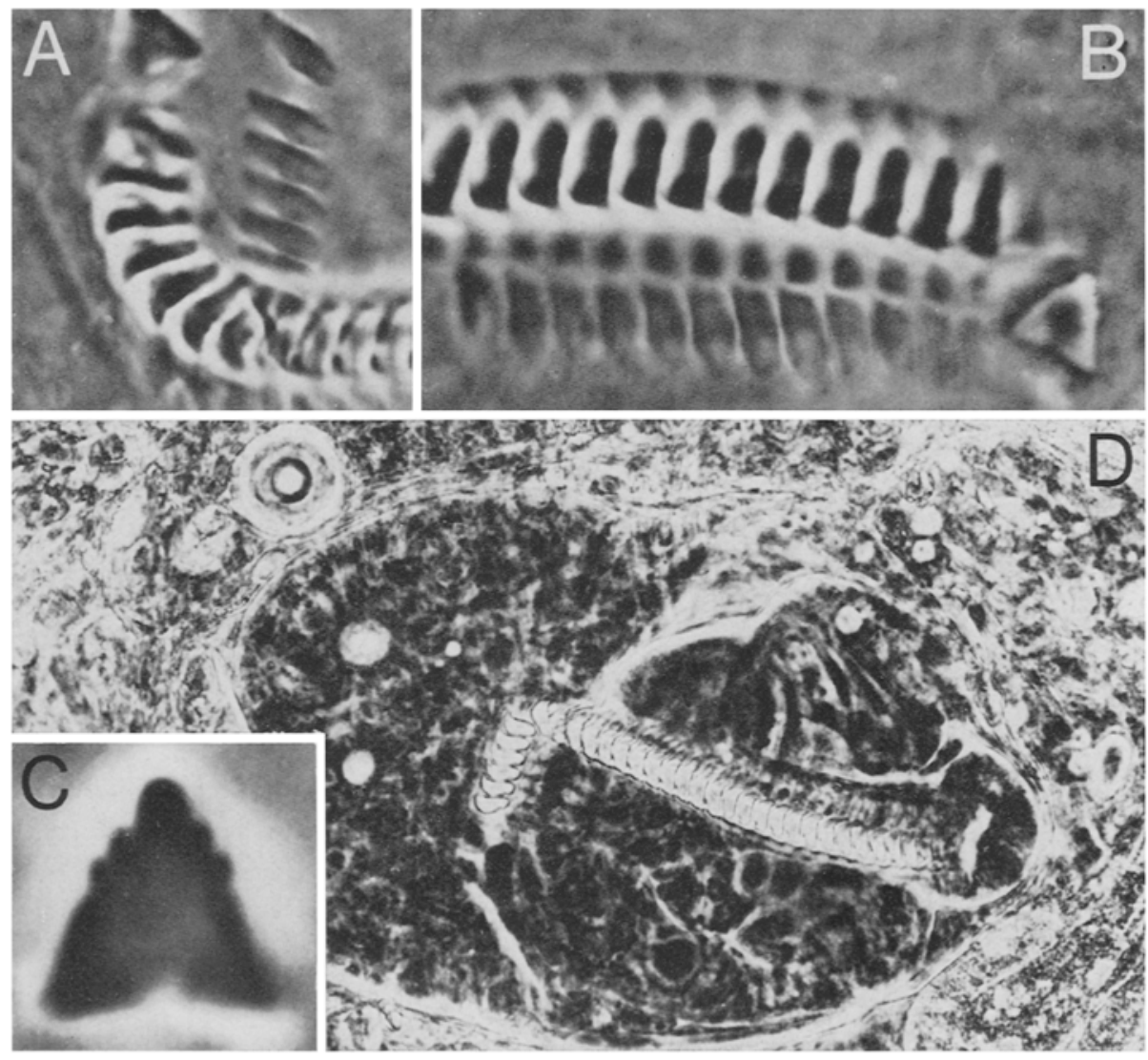

Abb. 6: Microbedyle cryptophthalma nov. spec. Radula. A Ausschnitt, Rhachiszähne mit linker Seitenzahnreihe; $B$ Ausschnitt, Rhachiszähne mit den beiden rechten Seitenzahnreihen; $C$ Rhachiszahn; $D$ Übersichtsphoto. (Alle Aufnahmen mit Phasenkontrast) 
je eine auffällige Statocyste mit einem Statolithen. Augen sind an lebenden Tieren und an Quetschpräparaten lebender oder fixierter Individuen nicht zu erkennen (siehe Fotos!). An aufgehelltem, fixiertem Material sieht man jedoch 2 winzige Pigmentflecke vor den Cerebralganglien.

Auffälligstes Organ ist die im Pharynxbulbus liegende Radula (Länge zwischen 60 und $110 \mu \mathrm{m}$ ) (Abb. 5,6). Sie besteht aus Zahnquerreihen, deren Zahl bei den einzelnen Tieren zwischen 26 und 36 differieren kann. Am häufigsten wurden Tiere mit 31 Reihen gefunden. 4 bis 9 (meist 6) der vorderen Zahnreihen sind nach ventral umgebogen. Geschlechtsreife und große Individuen besitzen zumeist auch die höchsten Zahnzahlen.

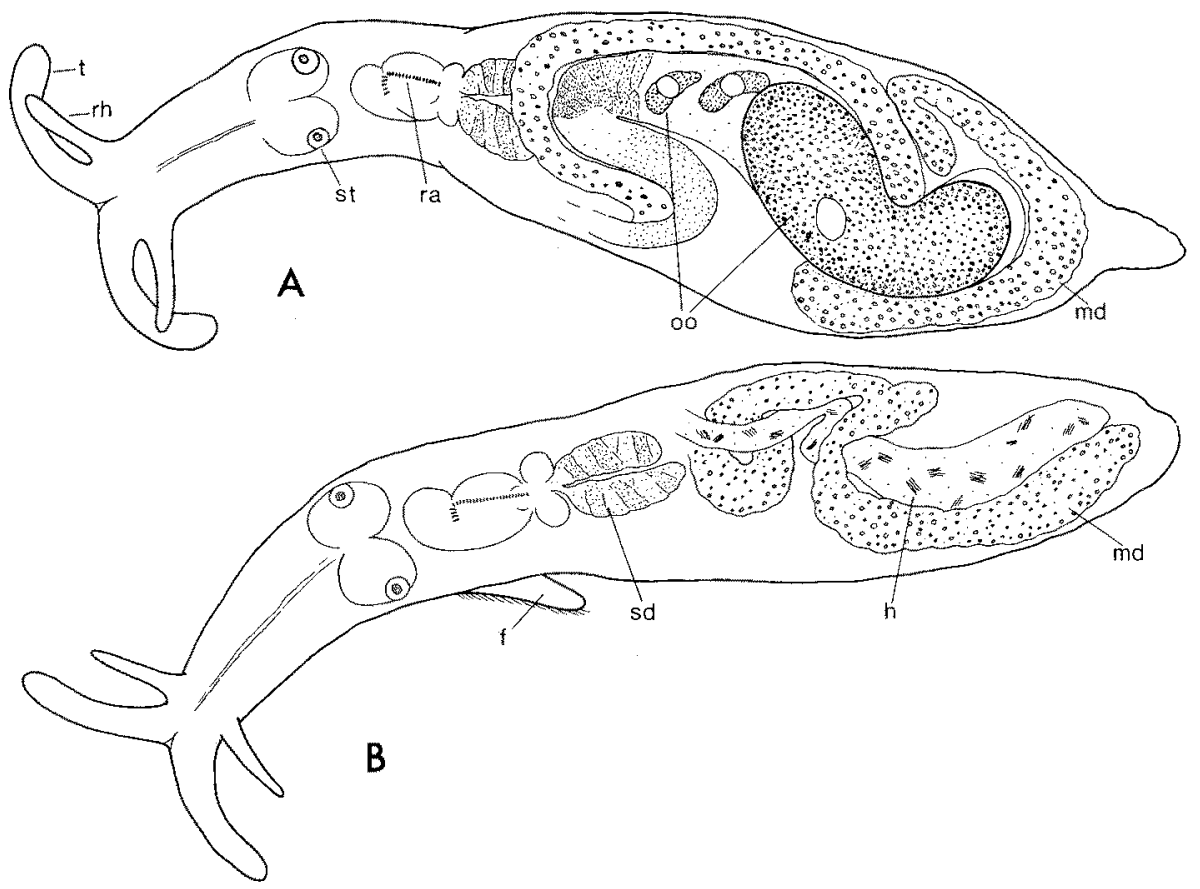

Abb. 7: Microhedyle cryptophthalma nov. spec. Freihandskizzen nach Quetschpräparaten. $A$ Weibchen; $B$ Männchen. $\mathrm{f}=\mathrm{Fuß}, \mathrm{h}=$ Hoden, $\mathrm{md}=$ Mitteldarmdrüse, oo = Oocyte, $\mathrm{ra}=$ Radula, $\mathrm{rh}=$ Rhinophor, $\mathrm{sd}=$ Speicheldrüse, $\mathrm{st}=$ Statocyste, $\mathrm{t}=$ Labialtentakel;

Augen wurden nicht eingezeichnet, da sie an lebenden Tieren nicht zu erkennen waren

Jede Querreihe besteht aus einem mittleren großen dreieckigen Zahn (Abb. 5, 6) und drei seitlichen Zahnplatten. Dies ergibt eine asymmetrische Radula mit 2 ungleich großen Elementen auf der rechten Seite und einer einzelnen Platte auf der linken. Der dreieckige Rhachiszahn besitzt an jeder Seite zwei Dentikel (Abb, 5B, 6C). Die Seitenplatten sind mehr oder weniger längliche, an den Edken leicht abgerundete Gebilde ohne Dentikel (Abb. 5D). Kiefer sind nicht vorhanden. Die Radulaformel kann daher mit 1.1.2. $\times \mathrm{n}$ angegeben werden. Die Zähne haben beim Typus folgende Maße: Rhachiszahn (auf der Seite liegend) $3 \mu \mathrm{m}$ breit, $6 \mu \mathrm{m}$ hoch; rechte äußere Platte $3 \mu \mathrm{m}$ breit, 
$9 \mu \mathrm{m}$ lang; rechte innere Platte $3 \mu \mathrm{m}$ breit, $6 \mu \mathrm{m}$ lang; linke Platte $3 \mu \mathrm{m}$ breit, $3 \mu \mathrm{m}$ lang.

Die Mitteldarmdrüse ist schlauchförmig und besitzt keine Divertikel. Das distale Ende ist nach vorn umgebogen; bei den meisten Tieren findet man eine doppelte, "spiralige" Umbiegung (Abb. 7A). Es konnten aber auch wenige Exemplare mit gerader Mitteldarmdrüse festgestellt werden (Abb. 7B).

Die Art ist getrenntgeschlechtlich. Es wurden insgesamt etwa 15 reife männliche und 4 weibliche Tiere gefunden.

Der Hoden ist ein einfacher schlauchförmiger Sack, der in den Windungen der Mitteldarmdrïse liegt (Abb. 7B). Er setzt sich nach vorn in einen breiten drüsenreichen Ductus ejaculatorius fort; die Geschlechtsöffnung liegt vor dem After auf der rechten Körperseite. Ein Penis ist nicht ausgebildet. Die Niere öffnet sich wahrscheinlich zwischen Geschlechtsöffnung und After nach außen. Die Spermien sind langgestreckte, fadenförmige Gebilde, die zur Hälfte spiralig gewunden sind (Abb. 9). Sie stimmen mit der Abbildung von Kow Alevsky (1901, pl. II, fig. 30) überein.

Das Ovar erstreckt sich ebenfalls zwischen den Windungen der Mitteldarmdrüse bis zum Hinterende. An Quetschpräparaten erkennt man einen durchsichtigen Sack mit einem großen, sehr dotterreichen Ei (Länge etwa $450 \mu \mathrm{m}$ ) (Abb. 9 A) und ein oder $z w e i$ kleineren Oocyten, deren Vitellogenese erst begonnen hat.

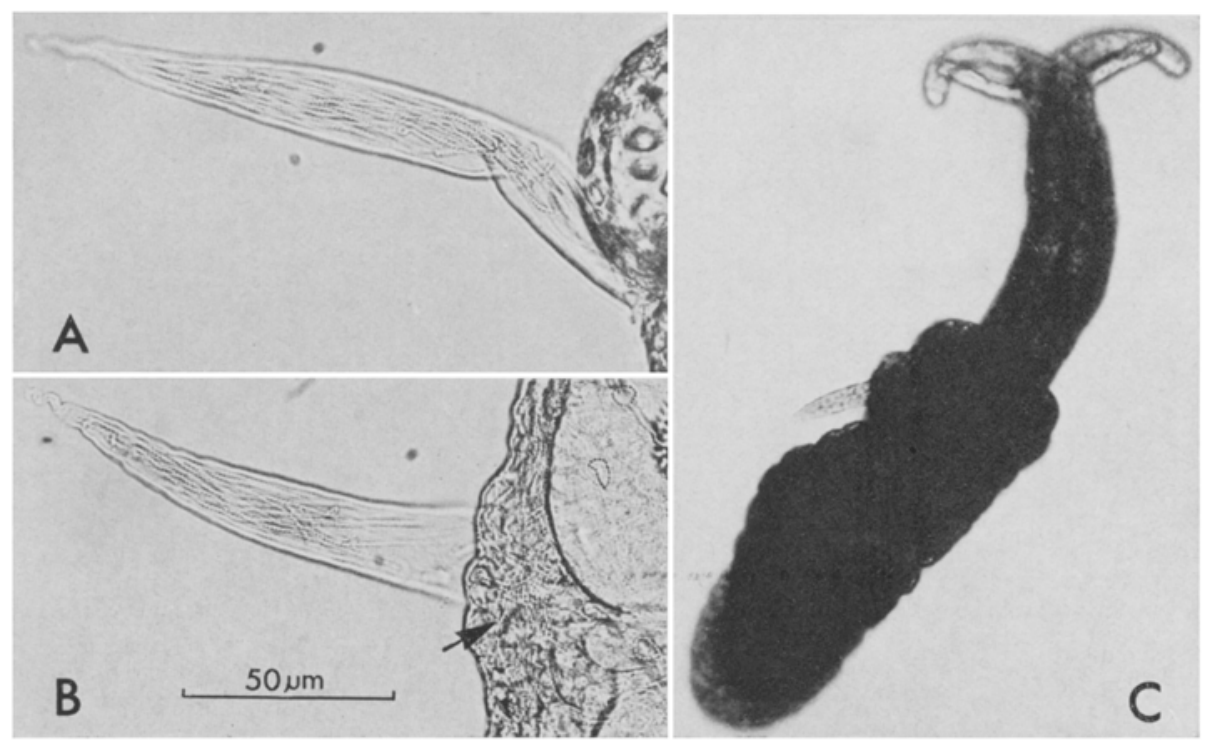

Abb. 8: Microbedyle cryptopbthalma nov. spec. $A, B$ Spermatophoren, Pfeil zeigt auf bereits in den Körper eingedrungene Spermien; $C$ Tier mit Spermatophore

\section{BIOLOGIE}

Geschlechtsreife Männchen wurden jeweils im September an der italienischen Küste (1970) und an der tunesischen Küste (1971) gefunden. Nur zu diesem letzteren 
Zeitpunkt konnten auch die wenigen Weibchen bei Nabeul und Amilcar nachgewiesen werden.

Die Spermaübertragung erfolgt durch Spermatophoren. Hierbei handelt es sich um keulenförmige Gebilde von unterschiedlicher Länge (80 bis $187 \mu \mathrm{m}$ ) und Breite $(17$ bis $30 \mu \mathrm{m})(\mathrm{Abb} .8,9)$. Sie bestehen aus einer dünnen durchsichtigen Hülle, die am distalen Ende in eine feine Spitze ausläuft. Im Inneren kann man etwa 200 bis 300 Spermien erkennen. In einem Fall hatten diese teilweise die Hülle verlassen und sich im Körper unter der Epidermis gesammelt (Abb. 8).

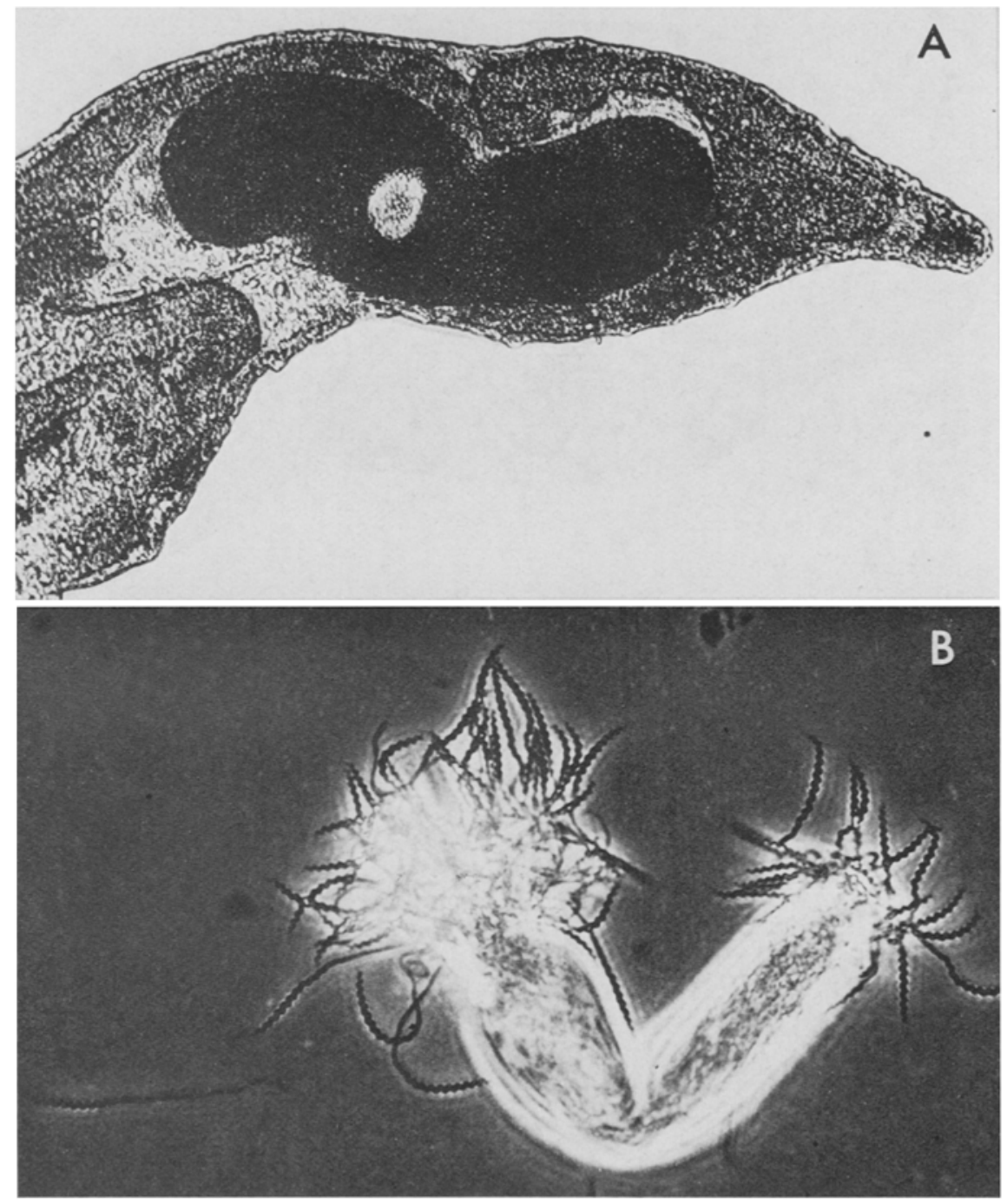

Abb. 9: Microbedyle cryptophthalma nov. spec. A Eingeweidesack eines weiblichen Tieres mit großer Oocyte; $B$ aufgeplatzte Spermatophore mit Spermien 
Insgesamt wurden acht Tiere aus der Population von Arco Felice mit derartigen Gebilden gefunden; zwei Individuen trugen leere Hüllen mit sich herum. Bis auf ein reifes Männchen handelte es sich um nicht geschlechtsreife Schnecken. Anheftungsstellen der Spermatophoren waren die lateralen Bezirke des Eingeweidesacks, zweimal auch das Vorderende in Höhe der Statocysten. Die Ubertragung konnte nicht beobachtet werden.

\section{DISKUSSION}

\section{System atik}

Nach dem Habitus, der Form der Kopfanhänge und der Mitteldarmdrüse, der Lage der Körperöffnungen und der Differenzierung der männlichen Geschlechtsorgane gehört die neue Art in die Familie der Microhedylidae (Ordnung Acochlidiacea, Opisthobranchia).

Form und Zahl der Radulazähne weisen sie eindeutig in den Formenkreis von Parbedyle, Microbedyle, Unela. Rein habituell (Besitz von zwei Paar Kopfanhängen, Ausbildung eines nur kleinen Fußes) stimmt $M$. cryptophthalma mit folgenden Arten aus diesen Gattungen überein: Parbedyle tyrtowii (Kowalevsky, 1900), Microbedyle glandulifera (Kow Alevsky, 1901), Microhedyle lactea Hertung, 1930, Microbedyle glomerans Salvini-Plawen, 1973, Unela remanei Marcus, 1953 und Unela odbneri (Delamare Deboutteville, 1953).

Microbedyle glomerans hat die gleiche Radulaformel wie die vorliegende Species, ist aber vor allem durch eine höhere Zahl der Rhachisdentikel und durch einen Dentikel an den Seitenplatten gut zu unterscheiden. Alle übrigen Arten differieren auch in der Radulaformel. $M$. glandulifera und $M$. lactea sind außerdem durch die Form ihrer Kalkeinschlüsse (Spicula) im Integument und den Besitz deutlich erkennbarer Augen von der neuen Art zu trennen. Weitere Differentialmerkmale von $M$. glandulifera sind die Pigmentierung der Kopf- und Speicheldrüsen sowie große Epidermisdrüsen, die in dieser Form bei $M$. cryptophthalma nicht vorhanden sind. Marcus \& Marcus (1954, p. 218) beschreiben eine von Remane gesammelte $M$. glandulifera ohne Augen. Der Fundort bei Neapel, vor allem aber Zahl und Form der Radulazähne machen es wahrscheinlich, daß den Autoren hier die neue Art vorgelegen hat. Die Gattung Unela besitzt im männlichen Geschlecht einen intraepidermalen Ductus, der zu den Rhinophoren führt (Marcus 1953) und kommt daher nicht für eine Einordnung in Frage.

Die größten Ubereinstimmungen bestehen zu Parbedyle tyrtoreii (KowaLEvskY 1900). Diese Form besitzt fast identische Hauteinschlüsse; die Größe ist ebenfalls vergleichbar. Eindeutig getrennt ist $M$. cryptophthalma auch von dieser Art durch die schwer erkennbaren Augen und die Form der Radulazähne: der Rhachiszahn besitzt bei jener Species vier seitliche Dentikel (Kowalevsky 1901, pl. III, Fig. 39), bei der neuen Art sind es nur zwei.

THIELE (1931) errichtete im 1. Band seines „Handbuch der systematischen Weichtierkunde" für diese Hedyle tyrtowii Kowalevsky die neue Gattung Parbedyle (p. 443). Im 2. Band des gleichen Werkes (1935, p. 1004) stellte er sie jedoch in das Genus Microbedyle, das bereits 1930 von HERTLING geschaffen worden war (vgl. BOETTGER 
1954). Der Gattungsname Parbedyle muß daher schon aus nomenklatorischen Gründen eliminiert werden. ODHNER (1952, p. 144) ignoriert dies. Er gibt vielmehr als Differentialmerkmale für Parbedyle an: die plattenförmigen Kalkeinlagerungen im Integument, das Fehlen großer Hautdrüsen und eine spiralig aufgewundene Mitteldarmdrüse. Eine derartige doppelte Aufwindung dieses Organs wird als Reminiszenz an Vorfahren mit noch spiralig gewundenem Eingeweidesack gedeutet. Nach dem vorliegenden Material ist dieses Merkmal aber nicht konstant genug, um eine Gattungstrennung $\mathrm{zu}$ rechtfertigen. Hierauf hat bereits MARcus (1953, p. 169) bei verwandten Arten hingewiesen. Auch die Form der Kalkelemente ist bei den Arten sehr variabel. Die diffizilen Merkmale „abgeplattete Tentakel“ und "große Epidermisdrüsen“ für Microbedyle und "zylindrische Tentakel" und "keine besonderen Epidermisdrüsen" für Parbedyle (MARcus 1953) reichen nach unserer Ansicht zur Aufrechterhaltung zweier Gattungen ebenfalls nicht aus. Parbedyle tyrtowii (Kowalevsky) gehört daher in die Gattung Microbedyle, ebenso wie die neue vorliegende Form. Diese Entscheidung ist völlig unabhängig von der Diskussion um die Zahl der Seitenzähne innerhalb der Gattungen Unela und Microbedyle und die daraus resultierenden systematischen Konsequenzen (Salvini-Plawen 1973).

Die weit auseinanderliegenden Fundorte der neuen Art an den tunesischen, italienischen, französischen und libanesischen Küsten deuten auf eine weite Verbreitung im Bereich des Mittelmeeres. Sie bestätigen die auch bei Polychaeten (Westreide 1972) nachgewiesene Gleichförmigkeit in der Zusammensetzung der interstitiellen Fauna in den sandigen Brandungszonen des Mittelmeeres. Die von Prof. Ax gesammelten Exemplare von Canet-Plage stammen vom Typlokal der Unela odhneri (Delamare Deboutteville) (Delamare Deboutteville 1953a, p. 352; 1953b, p. 419). Sie unterscheiden sich jedoch eindeutig von dieser Art, die bisher nur von Marcus \& Marcus (1955) untersucht wurde, durch Formel und Form der Radula. Es muß somit vorläufig angenommen werden, daß an diesem Ort zwei sehr ähnliche Vertreter der Acochlidiacea im Sandstrand nebeneinander yorkommen.

\section{Lebensformanalyse}

Geringe Körpergröße, Pigmentlosigkeit und sehr kleine Augen von Microbedyle cryptophthalma können als typische Lebensformmerkmale interstitieller Organismen betrachtet werden. Besondere Anpassungstmerkmale an den Biotop sind die geringe Gametenzahl und die direkte Spermaübertragung durch Spermatophoren (SWEDMARK 1964, Ax 1969). Innerhalb der Acochlidiacea sind Spermatophoren bisher bei Microbedyle lactea, Ganitus evelinae, M. milaschewitchii, $M$. glandulifera und Hedylopsis brambelli gefunden worden (MARCUS 1953, SwEDMARK 1968, WAWRA unveröffentlicht). Hedylopsis suecica dagegen besitzt spezifische Kopulationsorgane (ODHnER 1937), die einen anderen Modus der Ubertragung wahrscheinlich machen. Sehr ähnliche Spermatophoren, aber auch direkte Besamung durch hypodermale Injektion oder über weibliche Geschlechtsporen sind von interstitiellen Polychaeten bekannt (WEsTHErDE 1967, 1970). Damit ergeben sich interessante Konvergenzen in der Fortpflanzungsbiologie mesopsammaler Anneliden und Mollusken. 


\section{Artdiagnose}

Microbedyle cryptophthalma nov. spec. Bis 1,6 mm langer Körper, Breite des Eingeweidesackes bis $460 \mu \mathrm{m}$. Im Integument: ringförmige Einschlüsse ( 8 bis $9 \mu \mathrm{m}$ ) aus etwa 15 bis 20 kleinen Einzelgliedern; rundliche, plattenförmig erscheinende Einschlüsse, teilweise durchbohrt, mit mehr oder weniger zerklüfteten Umrißlinien; eiförmige, etwas größere, stark lichtbrechende Einschlüsse. Sehr kleine, schwer erkennbare Augen. Radulaformel 1.1.2. $\times \mathrm{n} ; \mathrm{n}=26$ bis 36. Mittlerer Radulazahn mit jeweils zwei kleineren Seitenzähnchen; dentikellose Seitenplatten, zwei auf der rechten, eine auf der linken Seite.

Der Typus ist das Quetschpräparat eines Exemplares von Nabeul (Tunesien). Es wird in der Mollusken-Sammlung des Naturhistorischen Museums in Wien unter der Nr. 79100 aufbewahrt; Paratypen befinden sich bei den Autoren.

\section{ZUSAMMENFASSUNG}

1. Microbedyle cryptopbthalma nov. spec. wurde im Mesopsammal verschiedener Sandstrände des Mittelmeeres (tunesische, italienische, französische und libanesische Küste) gefunden.

2. Die Tiere besiedeln eine schmale, besonders exponierte Region des Brandungsstrandes bis in eine Tiefe von $15 \mathrm{~cm}$. Eine Abhängigkeit der Besiedlung von den Sedimentverhältnissen konnte nicht festgestellt werden.

3. Verschiedene Merkmale der Organisation (Form der Einschlïsse im Integument, Radulaformel und Ausbildung der Zähne) weisen $M$. cryptophthalma als eine gut definierte neue Art aus.

4. Die Gattung Parhedyle wird eliminiert; die bisher einzige Art P. tyrtowii (KowALEVSKY) gehört in das Genus Microbedyle.

5. Die Spermaübertragung erfolgt durch einfache, spindelförmige Spermatophoren. Sie werden zusammen mit Merkmalen der Organisation als Lebensformcharaktere der Sandlückenfauna erkannt.

Danksagungen: Für dic Unterstützung der Aufenthalte am Mittelmeer dankt der Erstautor dem Mediterranean Marine Sorting Center in Khereddine (Direktoren: Dr. N. C. Hulings, Dr. R. P. Higgrns bzw. Dr. W. P. Davis), der Fritz-Thyssen-Stiftung, Köln, und der Akademie der Wissenschaften und der Literatur Mainz. Der Dank gilt weiterhin den Direktoren Dr. F. Ktarr bzw. Dr. A. Azouz und dem Personal vom Institut National Scientifique et Technique d'Océanographie et de Pêche in Salammbô sowie dem Personal der Stazione Zoologica in Neapel. Herrn Dr. B. Swedmark, Kristineberg, sei für wichtige Hinweise gedankt. 


\section{ZITIERTE LITERATUR}

Ax, P., 1969. Populationsdynamik, Lebenszyklen und Fortpflanzungsbiologie der Mikrofauna des Meeressandes. Verh. dt. zool. Ges. 62, 66-113.

Boettger, C. R., 1955. Die Systematik der euthyneuren Schnecken. Verh. dt. zool. Ges. 48, 253-280.

Challis, D. A., 1969a. An interstitial fauna transect of a Solomon Islands sandy beach. Phil. Trans. R. Soc. (B) 255, 517-526.

- 1969b. An ecological account of the marine interstitial Opisthobranchs of the British Solomon Islands Protectorate. Phil. Trans. R. Soc. (B) 255, 527-539.

- 1970. Hedylopsis cornuta and Microbedyle verrucosa, two new Acochlidiacea (Mollusca: Opisthobranchia) from the Solomon Islands Protectorate. Trans. R. Soc. N. Z. (Biol. Sci.) 12, $29-40$.

Delamare Deboutteville, C., 1953a. Recherches sur l'écologie et la répartition du Mystacocaride Derocheilocaris remanei Denamare et Chappurs, en Méditerranée. Vie Milieu 4, 321-380.

- 1953b. Description d'un appareil pour la capture de la faune des eaux souterraines littorales sous la mer. Premiers résultats. Vie Milieu 4, 411-421.

Hadi, G., Kothbauer, H., Peter, R. \& Wawra, E., 1970. Substratwahlversuche mit Microbedyle milaschewitchii Kowalevsky (Gastropoda, Opisthobranchia: Acochlidiacea). Oecologia $4,74-82$.

HerTLIng, H., 1930. Ober eine Hedyle von Helgoland. Wiss. Meeresunters. N. F. (Helgoland) $18,1-10$.

Kowalevsixy, A., 1900. Compte rendu de mes études zoologiques à Sebastopol. Jzv. imp. Akad. Nauk. 12, 193-204.

- 1901. Les Hedylidés. Etude anatomique. Zap. imp. Akad. Nauk (8. sér.) 6, 1-32.

Marcus, E., 1953. Three Brazilian Sand-Opisthobranchia. Bol. Fac. Filos Ciênc. Univ. S. Paulo (Zool.) 18, 165-203.

- \& Marcus, E., 1954. Uber Philinoglossacea und Acochlidiacea. Kieler Meeresforsch. 10, 215-223.

- - 1955. Uber Sand-Opisthobranchia. Kieler Meeresforsch. 11, 230-243.

ODHner, N. H., 1937. Hedylopsis suecica n. sp. und die Nacktschneckengruppe Acochlidiacea (Hedylacea). Zool. Anz. 120, 51-64.

- 1952. Petits Opisthobranches peu connus de la côte Méditerranéene de France. Vie Milieu $3,136-147$.

Porzat, C., 1971. Etude préliminaire des Gastéropodes Opisthobranches de quelques sables marines du Golfe de Marseille. Téthys 3, 875-896.

Salvini-Plawen, L. von, 1968. Neue Formen im marinen Mesopsammon: Kamptozoa und Aculifera (nebst der für die Adria neuen Sandfauna). Ann. naturh. Mus. Wien 72, 231-272.

- 1973. Zur Kenntnis der Philinoglossacea und der Acochlidiacea mit Platyhedylidae fam. nov. (Gastropoda, Cephalaspidea). Z. zool. Syst. Evolutionsforsch. 11, 110-133.

SChmidt, P., 1968. Die quantitative Verteilung und Populationsdynamik des Mesopsammons am Gezeiten-Sandstrand der Nordseeinsel Sylt. I. Faktorengefüge und biologische Gliederung des Lebensraumes. Int. Revue ges. Hydrobiol. 53, 723-779.

SWEDMARK, B., 1964. The interstitial fauna of marine sand. Biol. Rev. 39, 1-42.

- 1968a. The biology of interstitial Mollusca. Symp. zool. Soc. Lond. 22, 135-149.

- 1968b. Deux espèces nouvelles d'Acochlidiacées (Mollusques Opisthobranches) de la faune interstitielle marine. Cah. Biol. mar. 9, 175-186.

Thiele, J., 1931-1935. Handbuch der systematischen Weidhtierkinde. Fischer, Jena, 1-2, $1-1154$.

WESTHEIDE, W., 1967. Monographie der Gattungen Hesionides FRIEDRICH und Microphthalmus Mecznikow (Polychaeta, Hesionidae). Ein Beitrag zur Organisation und Biologie psammobionter Polychaeten. Z. Morph. Tiere 61, 1-159.

- 1970. Zur Organisation, Biologie und Ơkologie des interstitiellen Polychaeten Hesionides gobari Hartmann: Schröder (Hesionidae). Mikrofauna Meeresboden 3, 1-37. 
- 1972. La faune des Polychètes et des Archiannélides dans les plages sableuse à ressac de la côte méditerranéene de la Tunisie. Bull. Inst. natn. scient. tech. Océanogr. Pêche Salammbô (N. S.). 2, 177-196.

- \& Schmot, P., 1969. Von der Kleintierwelt im Meeresstrand. I. Fang und Untersuchung. Mikrokosmos 58, 257-262.

Anschrift des erstgenannten Autors: Dr. W. Westherde

II. Zoologisches Institut und Museum

Universität Göttingen

34 Göttingen

Berliner Straße 28

Bundesrevublik Deutschland 